\title{
Beam Tests on the ATLAS Tile Calorimeter Demonstrator Module
}

\author{
Eduardo Valdes Santurio ${ }^{\mathrm{a}, *}$, on behalf of the ATLAS Tile Calorimeter System., ${ }^{\mathrm{b}, *}$ \\ ${ }^{a}$ Stockholm University, Stockholm, Sweden \\ ${ }^{b}$ European Organization for Nuclear Research (CERN), Geneva, Switzerland
}

\begin{abstract}
The Large Hadron Collider (LHC) Phase-II upgrade aims to increase the accelerator luminosity by a factor of 5-10. Due to the expected higher radiation levels and the aging of the current electronics, a new read-out system of the ATLAS experiment hadronic calorimeter (TileCal) is needed. A Demonstrator prototype of the electronics has been tested during different testbeam campaigns at the Super Proton Synchrotron (SPS) accelerator of CERN with the purpose of checking the calibration and determining the performance of the detector by exploiting the features of the interactions of different particles with matter. We present the current status and results where the Demonstrator new electronics were situated in calorimeter modules and data were collected by exposing it to beams of muons, electrons and hadrons, at various incident energies and impact angles.
\end{abstract}

Keywords: HL LHC, Phase II upgrade, Demonstrator, Tile Calorimeter, ATLAS, TileCal, Testbeam,

\section{Introduction}

The High-Luminosity LHC (HL-LHC) will have an instantaneous luminosity of five times the nominal design value. ATLAS TileCal [1] (figure 1,) is a sampling calorimeter composed of steel plates and plastic scintillator tiles that is divided in four cylindrical barrels: the two central long barrel segments (LBA, LBC) and shorter extended barrel segments (EBA, EBC) at each end (figure 1p). Each cylindrical section is composed of 64 wedge-shaped modules where scintillators are grouped in pseudo-projective cells. Light from two sides of a cell is collected by wavelength shifting fibers and read out by two photomultiplier tubes (PMTs) (figure 17).

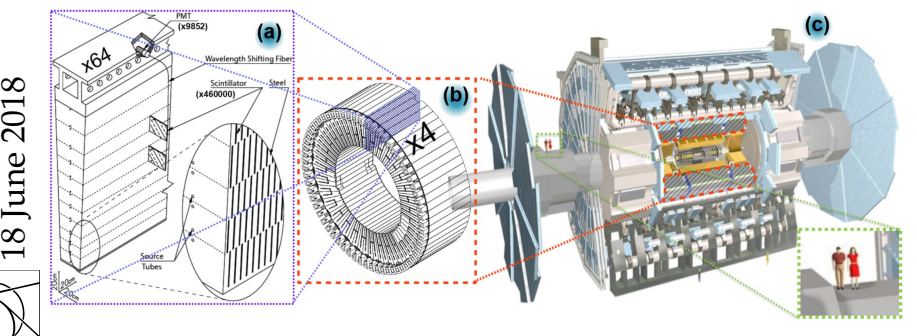

Figure 1: (a) A view of a TileCal wedge-shaped module [2]. (b) TileCal barrel[2]. (c) TileCal in ATLAS[1]

The Phase-II upgrade program of the ATLAS TileCal[2] will compensate for the higher rates of pileup, higher radiation levels and the aging of the current electronics. The read-out electronics will be replaced to adopt a fully-digital trigger system that will provide full-granularity digital data to the off-detector

\footnotetext{
${ }^{*}$ Corresponding author

Email address: eduardo.valdes@cern.ch, eduardo.valdes@fysik.su.se (Eduardo Valdes Santurio )
}

systems. The new design will provide better timing and energy resolution, and less sensitivity to out-of-time pileup. Protypes of the new electronics were coupled to calorimeter modules and tested in the Super Proton Synchrotron (SPS) at CERN. In order to check the calibration and to determine the performance of the detector, data were collected with beams of muons, electrons and hadrons of various incident energies and impact angles.

\section{The Phase-II and read-out system.}

The Phase II read-out system [3] is modularized in Superdrawers (SD), each composed of 4 Minidrawers (MDs). Frontend boards (FEB) interfaced with a Mainboard (MB) shape and condition the PMT signals. In every MD, a MB transfers to a Daughterboard (DB) the digitized 2 gains of 12 PMT signals at $40 \mathrm{Mhz}$. The DB interfaces the MB and the off-detector systems by receiving timing and slow control commands and transmitting data over redundant fiber-optic links (figure 2a). A Tile Preprocessor receives and stores two gains of PMT data from the DB in pipelines until a trigger decision event, while providing reconstructed data to the trigger. Given a trigger event, the data are read out by the FELIX system (figure $2 \mathrm{p}$ ).

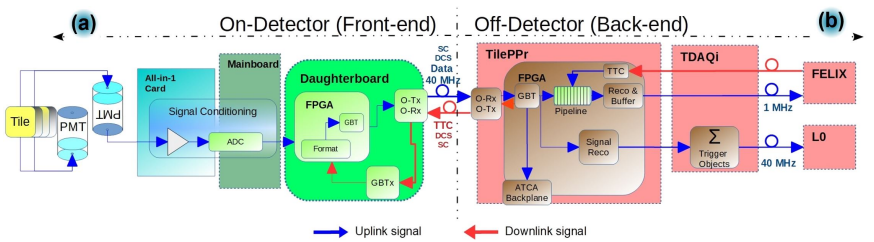

Figure 2: TileCal Phase-II Upgrade read-out system [3]. (a) On-Detector Electronics and (b) Off-Detector electronics 


\section{Testbeam setup and module layout on the scanning table}

The SPS H8 beamline is equipped with trigger scintillators in coincidence, Cherenkov counters for beam particle identification and wire chambers for measuring the lateral coordinates of the beam particles (figure 3 .

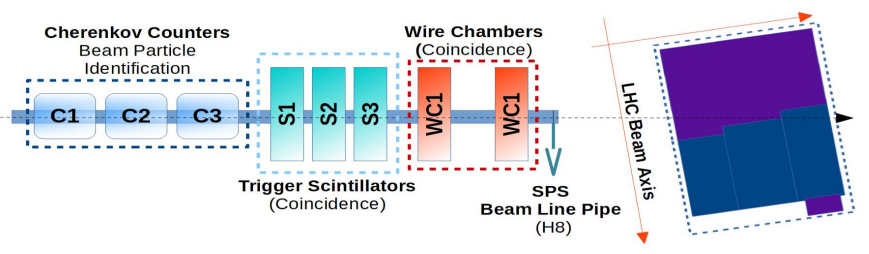

Figure 3: Modules and beam elements at the SPS H8 Beamline [4].

The testbeam setup accommodated 5 SDs [4] (figure 4): a demonstrator SD equipped with the 3-in-1 FE upgrade option in LBC 65, extra FE alternatives in a SD in LBA 65, two legacy SDs in M0 C and EBC 65, and Multi-Anode (MA) PMTs in a SD in M0 A for dedicated tests.

(a)

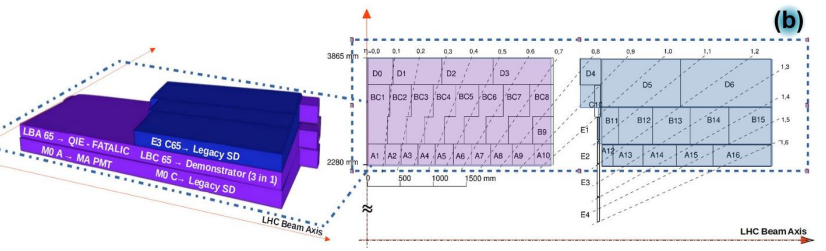

Figure 4: (a) Module layout on the scanning table [4] (b) Segmentation of LB and EB modules into cells. .

\section{Testbeam Results.}

Data taken from the Demonstrator module (LBC65) during the test-beam campaigns (2015-2017) was analyzed[4] [5]. The deposited muon energy followed a Landau distribution (figure 5 a). Studies were done for the "Fit" and "Optimal Filter" reconstruction methods with different noise thresholds (figure 5b). Cell responses were analyzed applying amplitude and timing cuts to the sums of the corresponding two PMTs signals (figure 5.).
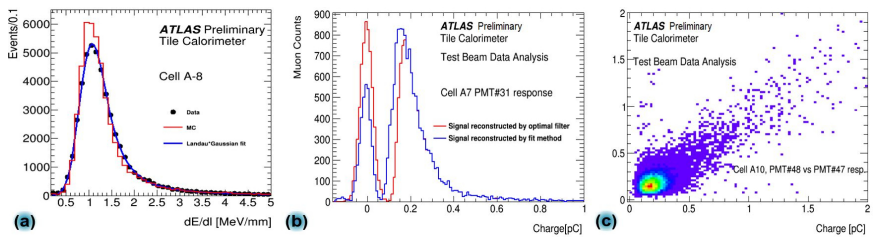

Figure 5: Muons results [4] [5]. (a) Distribution of $d E / d l$ for cell A8 using experimental data and simulated muons at $-90^{\circ}$. (b) $150 \mathrm{GeV} \mu$ reconstructed using Fit method and Optimal Filter hitting cell A7 at $-90^{\circ}$. (c) Correlation of signal responses in two PMTs of cell A10 (PMT48 vs PMT47).

The $e^{-}$energy distributions agree for both the Demonstrator and Legacy system (6a). The simulation and experimental data agree nicely for all studied energies (figures $6 \mathrm{~b}$ and 6 ).
The combination of the quantities $C_{\text {long }}$ (fraction of energy deposited in the first two longitudinal layers) and $C_{\text {total }}$ (spread of the energy deposited in the cell c) were used for $e^{-} /$hadron separation (figure 6d).
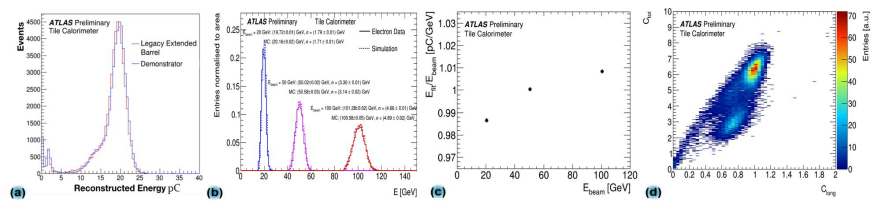

Figure 6: Electron results [4] [5]. (a) The cell response to electrons entering the calorimeter at $20^{\circ}$. (b) Total energy deposited in the calorimeter of 20 , 50 and $100 \mathrm{GeV} e^{-}$incident in the cell $\mathrm{A} 4$ at $20^{\circ}$ and simulated results using Geant 4.10.1 (FTFP_BERT). (c) Ratios $E_{\text {fit }} / E_{\text {beam }}$ for 20, 50 and $100 \mathrm{GeV}$ $e^{-}$incident in cell A4 at $20^{\circ}$. (d) The scatter plot of overall shower profile used for $50 \mathrm{GeV} e^{-} /$hadron separation hitting on cell $\mathrm{A} 4$ at $20^{\circ}$. The region on the right/top corresponds to electrons, the other to hadrons.

The hadron response was estimated using a Gaussian fit in the range $2 \sigma$ around peak value (figure $7 \mathrm{a}$ ). The analysis of the calorimeter response as a function of the beam energy for hadrons had good agreement with the simulation and resulted in higher response for pions and kaons than for protons, improving with increasing beam energy (figures $7 \mathrm{p}$ and $7 \mathrm{p}$ ).
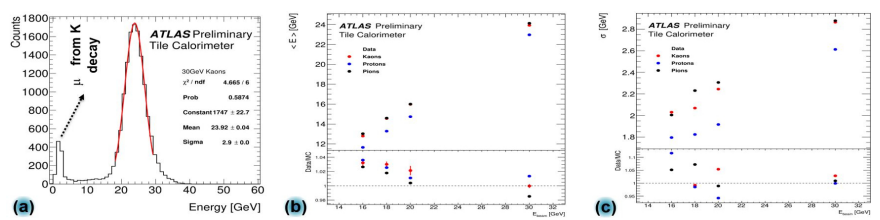

Figure 7: Hadron Results [4] [5]. (a) Energy distribution for $30 \mathrm{GeV}$ kaons. (b) Measured response for pions, kaons and protons as a function of the beam energy. (c) Measured width for pions, kaons and protons as a function of the beam energy.

\section{Conclusions}

Five testbeam campaigns of two weeks each took place between 2015 and 2017 with three detector modules resulting in a good performance of the new electronics, and agreement with calibration and simulated data. Two additional testbeams are scheduled for May and November 2018 following further integration with new revisions of the Phase-II upgrade hardware, mechanics and electronics.

\section{References}

[1] ATLAS collaboration. ATLAS tile calorimeter: Technical design report. CERN-LHCC-96-42, 1996

[2] E. Valdes, ATLAS Collaboration: Upgrade of Tile Calorimeter of the ATLAS Detector for the High Luminosity LHC, Journal of Physics: Conference Series, Volume 928, 2017.

[3] A. Valero, ATLAS Collaboration: Data acquisition and processing in the ATLAS tile calorimeter phase-II upgrade demonstrator, Journal of Physics Conference Series, Volume 898, 2017.

[4] T. Mkrtchyan, P. Pani, ATLAS Collaboration, ATLAS TileCal Performance Meeting report, ATL-TILECAL-SLIDE-2018-045, 2018

[5] ATLAS Collaboration, ATLAS Approved Plots https: //twiki.cern.ch/twiki/bin/view/AtlasPublic/ ApprovedPlotsTileTestBeamResults 